\title{
5 Estimating the CGE Model through the Maximum Entropy Principle
}

\subsection{Introduction}

In their reference work, Arndt et al. (2002) present a new approach to estimating parameters of a CGE model through maximum entropy. That approach is pursued here. CGE models are frequently criticised for resting on weak empirical foundations (e.g., Shoven and Whaley, 1992). Whatever class of CGE model is in use, it displays consistent drawbacks, such as the lack of efficient methodology for the estimates of behavioural parameters (e.g., trade parameters), less realistic economic assumptions (e.g., representative agent), less flexibility in implementing the monetary sector, imperfect competition, and the impossibility of inferring through interval confidence. In Social Accounting Matrix (SAM)-based CGE model estimation, problems related to calibration to a benchmark period, the often not updated information lying in the input-output matrix - the principal part of a SAM-and various other criticisms, appear in the economic literature. Nevertheless, as is often underscored there, the problem of estimates of behavioural parameters is common to competing time series econometric models unable to predict future agent behaviour (see the Lucas critique, 1976). Rational expectation-based models (Muth, 1961 and Kydland et al., 1977), like the dynamic stochastic general equilibrium models (DSGE) (Kydland et al., 1982), have tried to overcome the problem. Nevertheless, the DSGE models continue to display conceptual drawbacks from different sources, like the limited knowledge about the data generating system-and its future evolution-to which parameters are related (Evans and G. Ramey (2006), Tovar (2009)). Additionally, Sims (1987) reports additional drawbacks to the 'Lucas critique' in the context of rational expectations. Most of time, this may suggest that through the model with full information, we will deal with an over-parameterized, non-ergodic inverse problem, which traditional econometric approaches have failed to handle.

Next, in most developing countries, the alternative macro econometric models display serious weakness owing to scarce statistical data over large periods of time leading to technical problems of estimation (the degree of freedom) (Arndt, 2002).

Authors, such as Guerrien (2000) have expressed their scepticism about the usefulness of CGE models. Bernard Guerrien vigorously points to the less realistic economic assumptions such as the maximizing utility representative agent, perfect competition (Arrow-Debreu frame) or even imperfect monopoly markets; all these neo-classical concepts are described as fictive reality. The above criticisms have put into question the last strength of CGE models, that of being built on microeconomic foundations. Technical approaches to respond to most of the above criticisms have been attempted over the last two decades. After a thorough comparison between CGE and competing macro or microeconomic models (fixed-price models, dynamic 
optimisation/optimal control models, macro econometric models), authors such as Capros (Capros et al., 1990) suggest that all these models are complementary. More interestingly, the same authors provide useful insights into a future model that could address the cited shortcomings of current models.

Reconciling opposing models built upon contradictory assumptions would constitute too great a challenge. An acceptable solution should probably consist in setting up a model with only limited and realistic assumptions, basically combining common advantages of a CGE and a macro econometric equilibrium model.

This seems to be in line with two groups of authors in their recent works. One is by Robinson (Arndt et al., 2001, Go et al., 2015) who proposed a parameter estimation for a CGE using Shannon-Gibbs maximum entropy econometrics. These authors list additional qualities in comparison to the classical approach. One may cite the incorporation into the model of prior information related to present or past periods, thereby introducing dynamic elements into the system; the rationale for using entropy econometric formalism is the already cited quality of performing well in the absence of copious data. Last, the approach gives quantified information on the capacity of the model to reproduce a statistical record and computes statistical significance of parameter estimates.

The second work is by Francois (2001) who tries to overcome the problem of calibration: during the estimation process, the base period values are used to set up initial variable levels for the next steps of numerical estimation. However, numerical, successive approximations at the end can generate important deviations from the true values particularly when numerical processes imply multiplicative intermediary errors.

In this chapter, we try to extend the approach of Robinson (Arndt et al., 2002) to one of non-extensive entropy to estimate behavioural parameters for a CGE model.

The above authors have emphasized that the maximum entropy approach is similar to the econometric approach of Jorgenson $(1984,1998 \mathrm{a})$ in different aspects. To a certain extent, the full historical record can be employed and statistical tests for estimated parameter values are available. Furthermore, as pointed out in different works (e.g., Golan et al., 1996), the ME approach can be applied in the absence of copious data. The ME approach allows one to use all available data, take into account all relevant constraints, employ prior information about parameter values, and apply variable weights to alternative historical targets. Available information does not need to be complete or even internally consistent. The philosophy of the ME approach is to use all available information while avoiding the use of information not available, for example, strong assumptions about the distribution of error terms. 


\subsection{Estimation Approach}

In line with the preceding section, here the central question is why propose a maximum entropy econometrics related approach among many other econometrics techniques conceived for modelling large scale macroeconomic phenomena. On this point, it is worthwhile to refer, among a vast literature on the subject, to the review work of the Nobel laureate C. A. Sims (2007) on Bayesian methods applied in econometrics, in which he explains Why Econometrics Should Always and Everywhere Be Bayesian and then rejects frequentist asymptotics-based econometrics techniques. However, the above author did not allude to the maximum entropy econometrics approach as a competitive approach to Bayesian models ${ }^{51}$. According to Sims, the Bayesian approach uses the "Bayes rule" to incorporate the present beliefs (prior) about the phenomenon and to update this information with new facts at hand (data). Thus, following this author, unlike the frequentist-related econometrics methods, "Bayesian Inference is a way of thinking, not a basket of 'methods"” (Sims, 2007). ${ }^{22}$ It becomes interesting now to compare the Bayesian approach with the maximum entropy approach. Both approaches use priors and data to produce model posteriors. Nevertheless, beyond this similitude, the approaches are conceptually different.

Basically, applying Bayesian theorem means (Jaynes, 1988) just computing a probability and not a probability distribution. It follows that the Bayes theorem does not make any reference to sample space or hypothesis space. In empirical application of the Bayesian approach, we need to go beyond the "exploratory phase" to the point where a certain structure (the likelihood) of the model can be assessed through additional model distribution hypotheses.

On the contrary, the maximum entropy ${ }^{53}$ approach requires us to define a hypothesis space which sets down the possibilities to be further considered. Thus, in the exploratory phase of the problem, one can apply the entropy principle to solve a problem. In recent work, Giffin (2009) compared through illustrative computa-

51 Nevertheless, he pointed out that the Shannon mutual information approach has a more limited estimating capacity than the Bayesian one.

52 Jaynes [59] underscores the importance of this debate: "The recent literature has many attempts to clarify the relation of these principles.” Williams (1980) sees Bayes's theorem as a special case of MAXENT while van Campenhout \& Cover (1981) see MAXENT as a special case of Bayes's theorem. In our view, both are correct as far as they go, but they consider only special cases. Zellner (1987) generalizes Williams's results: "Thus Williams considers the case where we have a set of possibilities (H1 .. Hn), and some new information E confines us to a subset of them. Such primitive information can be digested by either Bayes' theorem or MAXENT, leading, of course, to the same result; but Bayes' theorem is designed to cover far more general situations. Likewise, van Campenhout \& Cover consider only the Darwin-Fowler scenario; MAXENT is designed to cover more general situations, where it does not make sense to speak of "trials"'.

53 To be more precise, we have in mind the method of relative Entropy (or cross-entropy) of which maximum entropy can be seen as a particular case. 
tions relative entropy, maximum entropy, and the Bayes rule in the environment of moments and data. Giffin underscored his finding related to the relationship between these three approaches. Only relative entropy can solve a problem by the simultaneous processing of data and moments-what Bayes and Maximum entropy alone cannot handle.

An interesting comparison between the Bayesian approach and the generalized maximum entropy approach (GME) is presented in Golan et al. (1996). As reported in these authors' work, Casella and Strawdermann (1981) used a simple example of recovering a bounded mean from a single observation $x=\beta+e$, where $e \sim^{`} N(0,1)$ and $\beta \subset[-c, c]$ are unknown to recover an image of $\beta$ from $x$. They first specified a discrete distribution with equal mass on points $-c$ and $c$, using the normal likelihood function and provided the Bayesian posterior mean-under the squared error loss-of the form:

$\hat{B}_{\beta}=c \tanh (c x)$

The above authors in Golan et al. (1996) performed the estimation of the same model using the generalized maximum entropy estimator and found the following GME solution:

$\hat{B}_{G M E}=c \tanh (-c \lambda)$

where $\lambda$ is the optimal Lagrange multiplier on the model constraint.

Authors showed that both estimators are related in mathematical formulation. Their estimates are equal only if $\lambda=-x$, which occurs when $x=0$. In this basic example, we notice that the Bayesian solution is directly influenced by the observation data $x$ while the GME solution, by the optimal Lagrange multiplier $\lambda$. Thus, the two formula illustrate not only the mutual consistency of both estimators but also a rich source of their confusion in scientific literature. The fact that the GME solution is a function of the optimal $\lambda$ is of high interest as this parameter is a function of the whole model, including the moments and the maximum entropy objective function. In the case of the generalized cross-entropy (GCE) technique, the advantage of that parameter for defining the optimal solution of a model is much more evident. In such a case, the optimal $\lambda$ displays a direct relationship with the prior, the data, the stochastic random disturbance, and the maximum entropy intrinsic properties. This may explain why the GCE approach seems to be preferred for solving stochastic ill-behaved inverse (non-ergodic) problems. To be more precise, the more constraining data are consistent with the model, the greater the value of $\lambda$ (absolute value), and the less uniform the maximum cross-entropy probability distribution. Unlike the Bayes approach, the GCE approach does not need any imaginative theoretical hypothesis to create a solution space closed-form. Golan (et al.) [61] comparing performances of different classes of econometric estimator in the case of ill-conditioned problems underscored the highest solution stability provided by the GCE technique. 
In fact, unlike other estimators, the penalty for using wrong prior information is much smaller relative to competitive estimators, including the Bayesian estimator. According to the above explanations, such superiority results from a diversified relationship between the optimal $\lambda$ and other constraints (data, stochastic random disturbance, and the maximum entropy objective function): the GCE estimates should not go far from the true parameters if only one constraint-in this case the prior-is violated. This result points to the GCE as the best estimation approach, at least in the case of stochastic ill-behaved inverse problems, as has been confirmed in a recent study (Bwanakare, 2014) which estimated parameters of three classes of constant elasticity of substitution models. The performance-measured on the parameter error variance coefficient-of the Tsallis related non-extensive cross-entropy estimator was much higher in comparison with the traditional econometric techniques (LS, ML, GMM, and NLS).

The number of authors who have tried to link CGE models with the cross-entropy econometrics approach is still limited (e.g., Arndt et al., 2002, Judge and Mittelhammer, 2012, and Go et al., 2015). In Arndt (2002) and Go et al. (2015), the authors start by viewing a classic, static CGE model in the following form:

$F(X, Z, B, \delta)=0$

where:

$F:$ an I-dimensional vector valued function,

$X$ : an I-dimensional vector of endogenous variables, usually prices and quantities,

$Z$ : a vector of exogenous variables such as endowments and tariff rates,

$B$ : a K-dimensional vector of behavioural parameters such as unknown constant elasticity of substitution parameters,

$\delta$ : a second vector of behavioural parameters whose values are uniquely implied by the choice of $B$, the exact form of $F$, and data for the base year.

The elements of $F$ capture production and consumption behaviour which is coherent in terms of economics as well as macroeconomic constraints. After parameter calibration and estimation, static CGE analysis proceeds by changing the vector of exogenous variables, $Z$, and examining through simulation the resulting vector of endogenous variables, $X$, which satisfies Equation (5.6).

In the entropy estimation formulation proposed by the above authors, the static model attempts to track the historical record over $T(t=1,2, \ldots, T)$ time periods. The $Z$ vector is partitioned into exogenous variables observable from historical data, $Z_{t}^{0}$, and exogenous variables not observable from historical data, $Z_{t}^{u}$. The vector $Z_{t}^{0}$ would typically contain historical data on elements such as tax rates, endowments, world prices, and government spending. The vector $Z_{t}^{u}$ might contain rates of technical change, implicit or unknown tax or subsidy rates. These variables and other items are not available from the historical record and must be estimated. Due to calibration to 
the base year and the restrictions imposed on the function, $F$, a unique relationship between $\delta$ and $B$ exists which permits the model in Equation (5.6) to reproduce the base year, conditional on the choice of behavioural parameters $B$,

$\delta=\Phi\left(Z_{t}, B\right)$

Note that the full vector $\mathrm{Zt}^{\prime}$ is assumed observable in the base year, labelled year $t^{\prime}$. Estimation occurs in the context of the CGE model, and then we have the next relationship:

$F\left(X_{t}, Z_{t}^{0}, Z_{t}^{u}, B, \delta\right)=0, \forall t \in T$

having to hold for estimated values $B$ and $Z_{t}^{u}$, imposed values $Z_{t}^{0}$, and calibrated values $\delta$. The outputs from such a solved problem lead to a predicted historical time path for variables of interest. These time series outputs can be compared with actual historic time paths in the following way:

$Y_{t}=G\left(X_{t}, Z_{t}^{0}, Z_{t}^{u}, B, \delta\right)+e_{t}$

where:

$Y_{t}$ : is an N-dimensional vector of historical targets defined inside the social accounting matrices,

$G$ : is a functional producing the vector of model predicted values for the targets,

$e_{t}:$ is an N-dimensional vector representing the discrepancy between historical targets representing the unknown data generating system $F$ and predicted values evaluated by the functional $G$, using sample information. Calibration to the base year implies that $e_{t}^{\prime}=0$.

After reparametrization of parameters $B, e_{t}, Z_{t}^{u}$, on defined support spaces according to the methodology explained earlier, the authors propose to set up a Gibbs-related cross-entropy (CE) model to be minimized under restrictions presented above. Since we specify prior distributions on parameters, the objective contains the two terms, precision and prediction (Golan et al., 1996), and each term can be given a weighting factor, $\alpha_{1}$ and $\alpha_{2}$. This CE formulation may be written as follows:

$$
\operatorname{Min} \underset{p, r}{\longrightarrow} \alpha_{1} \sum_{k=1}^{K} \sum_{m=1}^{M} p_{k m} \log \left(\frac{p_{k m}}{q_{k m}}\right)+\alpha_{2} \sum_{t=1}^{T} \sum_{n=1}^{N} \sum_{j=1}^{J} r_{t n j} \log \left(\frac{r_{t n j}}{s_{t n j}}\right)
$$

Subject to:

$$
\begin{aligned}
& F\left(X_{t}, Z_{t}^{0}, Z_{t}^{u}, B, \delta\right)=0, \quad \forall t \in T \\
& Y_{t}=G\left(X_{t}, Z_{t}^{0}, Z_{t}^{u}, B, \delta\right)+e_{t} \quad \forall t \in T \\
& \delta=\Phi\left(Z_{t}, B\right)
\end{aligned}
$$




$$
\begin{aligned}
& B_{k}=\sum_{m=1}^{M} p_{k m} v_{k m} \quad \forall k \in K \\
& e_{t n}=\sum_{j=1}^{J} r_{t n j} w_{t n j} \quad \forall t \in T, n \in N \\
& \sum_{m=1}^{M} p_{k m}=1 \quad \forall k \in K \\
& \sum_{j=1}^{J} r_{t n j}=1 \quad \forall t \in T, n \in N .
\end{aligned}
$$

Once again, the CE formulation in Equation (2.22) corresponds to the Kullback-Liebler measure of deviation of the estimated weights from the prior. The constrained optimization problem in Equations (2.23-2.24) chooses distributions for parameters and error terms that are closest to the prior distributions, using an entropy metric, and satisfies the full set of conditions required by a CGE model. In addition, the model endogenously calibrates itself to the base year.

The cited authors (Arndt et al., 2002) provide a case study on Mozambique.

Now, to extend the above approach to non-ergodic systems, we replace the objective function in (2.22) by the previously introduced criterion function of the form ${ }^{54}$ :

$$
\begin{aligned}
& \text { Min } \underset{p, r}{\longrightarrow} \alpha_{1} \frac{1}{q-1} \sum_{k} \sum_{m} p_{k m}\left[\left(p_{k m}\right)^{q-1}-\left(q_{k m}\right)^{q-1}\right]-\sum_{k} \sum_{m}\left(p_{k m}-q_{k m}\right)\left(q_{k m}\right)^{q-1}+ \\
& \alpha_{2} \frac{1}{q-1} \sum_{t} \sum_{n} \sum_{j} r_{t n j}\left[\left(r_{t n j}\right)^{q-1}-\left(s_{t n j}\right)^{q-1}\right]-\sum_{t} \sum_{n} \sum_{j}\left(r_{t t n j}-s_{t n j}\right)\left(s_{t n j}\right)^{q-1}
\end{aligned}
$$

subject to the same restrictions as above in (5.10).

In this case, we have then assumed a higher complexity of statistical data-generating system, and/or other kinds of systematic errors to which collected data might be prone. This case recalls characteristics of power law discussed in previous chapters. The value of the Tsallis parameter $q$ will inform us about the complexity of the system, as we already know.

The CGE outputs presented at the end of this chapter only limit entropy application to functions related to estimating behavioural parameters of constant elasticity of substitution. Further research on the methodology presented by the above authors could be of high interest. In particular, testing the proposed non-extensive relative entropy above, under the hypothesis of power law characteristics of macroeconomics remains urgent. In fact, in a recent publication, Bwanakare [62] has shown that trade functions used in CGE models may belong to the class of power law (Levy's process)

54 Note that there are two forms of Tsallis-Kullback-Leibler relative entropy. The one presented here comes from Bregman. Thus, it does not require escort distribution in the constraining block. 
distribution. The implication is that not taking this into account by the Shannonbased entropy could lead to significant estimation errors. To prove this, we are going to provide below an example showing that only estimation of trade parameters of a CGE model under power law hypothesis leads to adequate outputs.

\subsection{Application: Non-Extensive Entropy and Constant Elasticity of Substitution-Based Models}

The example below shows the possibility of carrying out robust estimation of a stochastic constant elasticity of substitution (CES)-based model through the Tsallis entropy econometrics technique. The estimator properties for Tsallis entropic form have been suggested in Part II of this book, in the section devoted to the parameter confidence interval area-based statistical inference.

The technique presented below has been suggested by Bwanakare (2014) or more recently in Bwanakare (2016). This extended the results through the case study of eight CES production (CESP) models of seven countries. While the proposed approach could be generalized to a large class of nonlinear models, the example focuses on CGE trade models, the parameters of which are usually obtained through a calibration technique. The CES-based models remain intractable while trying to analytically estimate their parameters. In empirical research, various nonlinear approximation techniques, like the k-th order Taylor polynomial technique, are applied and completed by approaches that use least square methods. As will be shown below, such approaches do not conceptually fit this type of non-ergodic model and estimated parameters should remain biased and inefficient. In this document, we suggest a power law (PL)driven estimation approach, thus moving away from a Gaussian ergodic hypothesis to more general Levy, unstable time (or space) processes, characterized by tail queues, long memory, complex correlation, and plausible convergence to the Gaussian central theorem limit. Once again, as in the case of a labour demand model presented at the end of Part II, the estimation procedure presented could be seen as a generalization to non-ergodic systems from the work of Kullback-Leibler on information divergence (Kullback, 1951 and Golan et al., 1996) on entropy econometrics. Technically, we minimize the Tsallis non-extensive relative entropy criterion function under consistency moment-constraints-incorporating the reparameterized CES function-and regular normality conditions. As such, the approach then encompasses the Bayesian information processing rule while remaining, however, fundamentally based on the second law of thermodynamics ${ }^{55}$.

55 For those interested, a vast literature on the subject can be found at: http://polymer.bu.edu/ hes/ econophysics/ 


\subsubsection{Power Law and the Constant Elasticity of Substitution (CES) Function}

A half century ago, Arrow, Chenery, Minhas, and Solow (1961) proposed a new mathematical function which simultaneously displays the property of homogeneity, constant elasticity of substitution (CES) between factors of production. Additionally, this function presents the possibility of differentiating elasticity of substitution for different industries, sectors, or countries (Klump and Papageorgiou, 2008), thereby generalizing the Cobb-Douglas model. The model was later expanded to other case studies where the system optimally aggregates its components according to some parameters to be specified below. Following Bwanakare (2014), we develop the proposed entropy formalism using a classical CESP explaining the gross domestic product $\left(V A_{t}\right)$ by two classical factors: labour $\left(L_{t}\right)$ and capital $\left(K_{t}\right)$.

The next two cases of the CGE trade model class have been presented in Bwanakare (2014). Let us recall below a CESP mathematical form:

$V A_{t}=\alpha\left[\partial K_{t}^{-\rho}+(1-\partial) L_{t}^{-\rho}\right]^{-\frac{v}{\rho}} e^{\varepsilon_{t}}$

or one of its generalized formulations as:

$Y=\left[\sum_{i=1}^{n} a^{(1-\rho)}{ }_{i} X_{i}{ }^{p}\right]^{-\frac{v}{\rho}}$

where:

$\rho=\frac{1-\tau^{e}}{\tau^{e}}$ with $-1 \prec \rho \prec+\infty$ and $0 \prec \tau^{e} \prec+\infty$

and

$\tau^{e}$ constant elasticity of substitution between factors, $\varepsilon_{t}$ stands for the random disturbance with unknown distribution. In (5.12), $\alpha$ stands for the shift parameter; the parameter $\delta$ belongs to the zero-one interval and represents the share (distribution) of the sold quantities of both distributed factors. Parameter $v$ reflects the degree of changing returns of $V A_{t}$ to scale. The higher the value of $\rho$, the higher the degree of substitution between factors. The case of $\tau^{e}$ converging to 0 suggests perfectly substitutable factors. The generalized form (5.13) suggests a case of more than two inputs $X_{i,} i=1,2, \ldots, n$.

Let us now focus on a useful connection between the CES class of functions and a power law (PL). In fact, to better display that relation, let us aggregate components of model (5.12) into one variable. Then, we get a generic case of a PL of the form:

$v a_{t}=\alpha\left[\lambda k_{t}^{-p}+1\right]^{-\frac{v}{\rho}} e^{\varepsilon_{t}}=\beta k_{t}^{\eta} e^{\varepsilon_{t}}$

where, in this case, the endogenous variable $v a_{t}$ is the product per capita. Parameter $\beta$ represents a general level of technology. The variable $k_{t}$ stands for a capital coefficient. 
The exponent $\eta$ belongs within the interval $(-1,+\infty)$ and defines a per head product elasticity with respect to the capital coefficient. It is evident that the above relation (5.15) can be compared with the relation (2.30) or the achieved tail queue-related Tsallis non-extensive entropy relation (2.31). The random term $\varepsilon_{1}$, itself, is assumed to follow PL structure. Index $t$ means time period. Thus, such PL relationships with the class of CES function seem to engender potential implications on economic grounds, including extensions to the real demand side of the economy. A PL displays interesting properties which may explain its ubiquity at different complexity levels in natural and human organizations. For a survey on the inheritance mechanism and other properties of a PL (Gabaix, 2008). Once again, for the direct relationships between a PL and non-extensive Tsallis entropy, refer to Tsallis (2009). The proposed model generalizes the statistical theory of information approach to non-ergodic systems where $q$ is different to unity. Let us underscore the fact that many findings of recent decades seem to confirm a domination of such systems in the physical real world. Even if we do not intend to set up a philosophical discussion here, a large number of econo-physical contributions of recent years seem to confirm the ubiquity of nonergodic law in social sciences (e.g., Bottazi et al., 2007, Ikeda et al., 2008, Mantagna et al., 1999). Under such a hypothesis, this could constitute a serious drawback for other competing entropy or econometrics techniques when trying to efficiently model a certain class of phenomena.

The model estimation. We follow the same procedure as in previous examples when we searched to minimize the cross-entropy criterion function under a priori moments, including the one concerning the economic model in question. Below, we directly present the model under the reparameterized form of parameters. Then, if we additionally use an escort distribution in moments, the Tsallis cross-entropy econometric model can be stated as:

$$
\operatorname{Min} \underset{a, p, r}{\longrightarrow} \alpha\left[\sum a_{j m} \frac{\left[a_{j m} / a o_{j m}\right]^{q-1}-1}{q-1}+\ldots .+\sum p_{k m} \frac{\left[p_{k m} / p o_{k m}\right]^{q-1}-1}{q-1}\right]+\beta \sum r_{n j} \frac{\left[r_{n j} / r o_{n j}\right]^{q-1}-1}{q-1}
$$

s.t.:

$$
\begin{aligned}
& \ln V A=\ln \left(\sum_{j=1}^{J} g_{j} \frac{a_{j}{ }^{q}}{\sum_{j=1}^{J} a_{j}{ }^{q}}\right)-\frac{\sum_{h=1}^{H} v_{h} \frac{w_{h}{ }^{q}}{\sum_{h=1}^{H} w_{h}{ }^{q}}}{\left(\sum_{m=1}^{M} v_{m} \frac{p_{m}{ }^{q}}{\sum_{m=1}^{M} p_{m}{ }^{q}}\right)} \ln \left[\sum_{i=1}^{I}\left(t_{i} \frac{b_{i}{ }^{q}}{\sum_{i=1}^{I} b_{i}{ }^{q}}\right) L{ }^{\left(\sum_{m=1}^{M} z_{m} \frac{p_{m}{ }^{q}}{\sum_{m=1} p_{m}{ }^{q}}\right.}+\left(1-\sum_{i=1}^{I}\left(t_{i} \frac{b_{i}{ }^{q}}{\sum_{i=1}^{I} b_{i}{ }^{q}}\right) K^{\left(\sum_{m=1}^{M} z_{m} \frac{p_{m}{ }^{q}}{\sum_{m=1}^{M} p_{m}{ }^{q}}\right)}\right]+\right. \\
& +\sum_{n=1}^{N} \sum_{j=1}^{J} z_{n j} \frac{r_{j}{ }^{q}}{\sum_{j=1}^{J} r_{j}{ }^{q}} \\
& \sum_{j>2 \ldots M}^{J} a_{j}=1 \sum_{m>2 \ldots M}^{M} p_{m}=1
\end{aligned}
$$


$\sum_{i>2 . . I}^{I} b_{i}=1$

$\sum_{h>2 . . I}^{H} w_{h}=1$

For reasons of formal presentation, the criterion function (Equation 5.16) does not include probabilities $w_{h}$, explaining degree of economy changing to scale and $b_{i}$, the parameter of distribution between factors.

In order to improve the estimated parameter quality-in the Bayesian senseadditional constraining data can be added to (5.17-5.20). In the case of a CES model, some economic theory exists. For instance, we can predict sign value domain variation for each parameter. Then we get:

$0 \leq \alpha=G a<\infty$

$-1 \leq \rho=Z p \leq \infty$

$0 \leq \delta=T b \leq 1$

Where $\alpha, \rho, \delta$ in Equation 1a stand for the original, "before-reparameterization," parameters. The set $G, Z, T$ stand for the above original parameter support space with the corresponding weight-probabilities $a, p, b$ defining output posteriors. $G, Z$, $T$ support spaces are defined in the same way as, e.g., Equation 2.45-2.46. Here we just present how we have specified this particular model and not a general rule of specification. Note that depending on error distribution, the weights $\alpha, \beta$ introduced in the above dual objective function may exercise a significant impact on the model optimal outputs, respectively, as precision and prediction weight. Indeed, the entropy model encompasses statistical losses in the parameter solution space (precision) and in the sample solution space (prediction). As can be easily shown, Lagrange multipliers stand for implicit nonlinear function of the weights $(\alpha, \beta)$ imposed in the generalized cross-entropy criterion function. Changes in weights alter the corresponding optimal solution value. In general, as in most constrained optimization problems, smaller Lagrange multipliers for a $q$ cross-entropy formulation imply smaller impact of constraints on the objective, in particular for the Tsallis $q$ around unity, i.e., the Gaussian case.

Model outputs. Outputs presented below constitute an important component of the findings in this book. They underscore, more than in previous applications where, generally, outputs from Tsallis entropy fit with those of Shannon entropy, i.e., an illustration of convergence case to Gaussian model. Here, things will change and power law will point out its form. Because of its importance, many details of the model outputs already presented by Bwanakare (2014) will be reported in this section plus new outputs from two additional country case studies. Thus, based on the data source of Table 22, let us present outputs of the three CGE trade models: the produc- 
tion function CESP (already developed through the above sections), the CECS (constant elasticity of commercial substitution function, known as the Armington model), and the CET (constant elasticity of technical transformation). The reasons for presenting these three models of the same class of function are the following: the CES model displays causality relationships; the CECS remains a quasi-identity equation since it is just missing a quasi-constant variable (the indirect taxes) to constitute an identity; and the CET model remains an identity equation, the covariate values of which sum up to the explained value of the model. Statistical data in Table 21 illustrate that situation. Due to a low level of precision, the traditional regression techniques may not be relevant in separating the three cases presented above. We compare the outputs from non-extensive cross-entropy (NCEE) with those from the traditional estimation techniques: the nonlinear least squares (NLS), the generalized methods of moments (GMM), and the maximum likelihood approaches (Green, 2003). Model data first have been dimensioned at logarithmical scale for computational purposes. The computations of the NCEE model were carried out with the GAMS code (General Algebraic Modelling System). Those with the NLS technique were done with Microsoft Excel. Computations by the GMM and ML approaches were executed with a special code from the open source GRETL. Let us first recall the mathematical formulation of the next two CES model classes. A CECS function aggregating interior economic absorption with two business components (locally produced commodities demand $(D O)$ and imports $(M)$ has the following analytical form:

$$
C_{t}=\alpha\left[\partial D O_{t}^{-\rho}+(1-\partial) M_{t}^{-\rho}\right]^{-\frac{1}{\rho}}
$$

where:

$$
\rho=\frac{1-\tau^{e}}{\tau^{e}} \text { with }-1 \prec \rho \prec+\infty \text { and } 0 \prec \tau^{e} \prec+\infty
$$

and

$\tau^{e}$ constant elasticity of substitution, $\varepsilon_{t}$ stands for random disturbances with unknown distribution.

The last model CET is analytically formulated in the following way:

$$
M O_{t}=\alpha\left[\partial e x_{t}^{-\rho}+(1-\partial) D M O_{t}^{-\rho}\right]^{-\frac{1}{\rho}}
$$

where:

$$
\rho=\frac{1-\tau^{e}}{\tau^{e}} \text { with }-\infty \prec \rho \prec-1 \text { and }-\infty \prec \tau^{e} \prec 0
$$

and

$e x_{t}, D M O_{t}$ stand for exports and domestically marketed outputs, respectively. The rest of the symbols have the same meaning as in the previous models. The higher the value 
of $\rho$, the higher the degree of transformation. When that parameter converges to $-\infty$ we are dealing with a case of perfectly complementary products, which refers to Leontief technology. The case of $\rho$ converging to -1 suggests perfectly substitutable products. In this study, priors were initiated from NLLS outputs. As known, such priors are not deterministically fixed. They are updated according to the Bayesian information processing rule. For simulation purposes, different $q$-Tsallis parameter values were computed from unity to its admissible ${ }^{56}$ highest values minimizing the criterion function. In all models, an a priori parameter support space for reparameterization varies between -5.0 and +5.0 . The same prior space has been retained for the error disturbance with amplitude varying between -3 and +3 , so it conforms to the three sigma rule owing to Chebychev's inequality (Pukelsheim, The Three Sigma Rule, 1994). Both spaces are symmetric around zero. This prevents the estimated parameters from a bias. All the recent works on these subjects (Bwanakare, 2016) seem to confirm that besides the NCEE approach, the NLS remains much better than the remaining econometric methodologies (GMM and ML techniques) for solving this kind of nonlinear inverse problem. Then, in the next model we limit ourselves to the presentation of the model outputs from the NCEE and NLS techniques. Tables 18 and 19 comparatively display the outputs from NCEE and NLS, respectively, for the three models. The NCEE estimator super-consistency for all three models can be noticed despite the small sample. The NLS approach seems better than the GMM procedure as shown in, e.g., Bwanakare (2016). The ML has produced, as theoretically expected, much poorer outputs. Output performance is displayed through error curves in Figures 4 to 9.

\subsubsection{Parameter Outputs of the Tsallis Relative Entropy Model}

Nonlinear LS estimation outputs. Using traditional nonlinear least square methods, we have linearized the Equations (1a, 1c, and 1d) before applying the Taylor development and the LS approaches.

The NCEE outputs are accurate for all estimated models, and performances of the rest of the econometric approaches seem to be much less competitive. Having used a twelve-year sample in this model, the power law clearly seems to constitute the data generating system. Let us thus comment on these NCEE outputs on the empirical side. The estimated parameters reflect long-run optimal equilibrium values of the system. Since we are dealing with the aggregated accounts of 27 EU countries, the values of estimated parameters seem to reflect our expectations. In particular, in the case of the CESP production model, the estimated parameter $p$ with an estimate

56 In fact, its interval covers Gaussian $(1 \prec q \prec 5 / 3)$ and stable laws (e.g., Levy's) attractors for $(5 / 3<q<3)$. 
Table 18: Outputs from the NCEE: dependent var: $C(t), M O(t), V A(t)$.

\begin{tabular}{lllllll}
\hline Exogenous var: & $\boldsymbol{A}$ & $\boldsymbol{\delta}$ & $\boldsymbol{p}$ & $\boldsymbol{v}$ & $\boldsymbol{I}(\boldsymbol{P r})$ & $\boldsymbol{C} \boldsymbol{V}$ \\
\hline $\mathrm{CESP}(\mathrm{L}(\mathrm{t}), \mathrm{K}(\mathrm{t}))$ & 1.866 & 0.163 & 0.001 & 1.000 & 0.99 & 0.006 \\
\hline $\mathrm{CET}(\mathrm{DO}(\mathrm{t}), \mathrm{Ex}(\mathrm{t}))$ & 2.000 & 0.5 & -1.0001 & & 0.999 & $4.271 \mathrm{E}-7$ \\
\hline $\mathrm{CECS}(\mathrm{C}(\mathrm{t}), \mathrm{M}(\mathrm{t}))$ & 2.000 & 0.499 & $\mathrm{pu}-0.985$ & & 0.999 & $2.705 \mathrm{E}-5$ \\
\hline$q$ Tsallis parameter (weight $\left.\alpha_{\mathrm{i}}=0.05\right)=2.333$ (CECP) & & & \\
$q$ Tsallis parameter (weight $\left.\alpha_{\mathrm{i}}=0.05\right)=1.0001$ (CECS) & & & \\
$q$ Tsallis parameter (weight $\left.\alpha_{\mathrm{i}}=0.05\right)=1.0001$ (CET) & & & \\
\hline
\end{tabular}

Table 19: Outputs from the NLS, models CESP, CET, CECS: dependent var: $C(t), M O(t), V A(t)$

\begin{tabular}{llllll}
\hline Exogenous var: & $\boldsymbol{A}$ & $\boldsymbol{\delta}$ & $\boldsymbol{p}$ & $\boldsymbol{v}$ & $\boldsymbol{R}^{2}$ \\
\hline CESP(L(t), K(t)) & 1.995 & 0.282 & 3.046 & 0.993 & \\
\hline Parameters T-value) & 48.89 & 6.61 & 1.49 & 6.61 & 0.88 \\
\hline CET(DO(t),Ex(t)) & 2.008 & 0.497 & -0.954 & & \\
\hline Parameters T-value & 558.120 & 1551.353 & -292.532 & & 0.999 \\
\hline CECS (C(t), M(t)) & 2.147 & 0.477 & -0.532 & & \\
\hline Parameters T-value & 6.257 & 13.073 & -1.554 & & 0.83 \\
\hline
\end{tabular}

Table 20: Outputs from the NCEE: dependent var: GDP(t)

\begin{tabular}{lllllll}
\hline COUNTRY & $\boldsymbol{A}$ & $\boldsymbol{\delta}$ & $\boldsymbol{p}$ & $\boldsymbol{Q}$ & $\boldsymbol{E}$ & $\mathbf{n}$ \\
\hline Belgium & 2.326 & 0.15 & $-3.524398 \mathrm{E}-77 / 3$ & 0.027 & 18 \\
\hline USA_sic33 & 0.777 & 0.057 & $-9.84248 \mathrm{E}-5$ & $7 / 3$ & $3.373000 \mathrm{E}-4$ & 20 \\
\hline
\end{tabular}

around zero suggests a convergence of the analysed function to the classical CobbDouglas function, displaying in the present case constant returns to scale. A long-run optimal equilibrium share parameter $\delta$ between factors shows a lower proportion of labour of around $16.3 \%$ with respect to capital share (83.7\%). In 2010, this proportion was around $57 \%$ for labour. Thus, according to these outputs, long-run optimal production will require much less labour demand, around 16\%. For the models CET and CECS, the estimated parameters show, in the long-run, a quasi-perfect substitutability and a balanced share between local and foreign commodities. Expected free trade 
Table 21: Outputs from the NLS: dependent var: GDP(t)

\begin{tabular}{llllll}
\hline Exogenous var: & $\boldsymbol{A}$ & $\boldsymbol{\delta}$ & $\boldsymbol{p}$ & $\boldsymbol{v}$ & $\boldsymbol{R}^{2}$ \\
\hline Belgium & 11,85713 & 1,82255 & 2,833935 & 0,867049 & 0.999 \\
\hline USA_sic33 & 0,00038 & 4,85717 & 0,061815 & 1,054080 & 0.999 \\
\hline
\end{tabular}

Table 22: Aggregated data (chain-linked volumes at 2005 exchange rates) for models (in 1000 billion euro).

\begin{tabular}{|c|c|c|c|c|c|c|c|c|c|c|c|c|}
\hline year & 999 & 2000 & 2001 & 2002 & 2003 & 2004 & 2005 & 2006 & 2007 & 2008 & 2009 & 2010 \\
\hline$V$ & & & & & & & & & & & & \\
\hline$d$ & & & & & & & & & & & & \\
\hline t & 4 & 4 & & & & & & 5 , & & 5 & 5,691 & \\
\hline MO & 691 & 9,026 & 9 & 25 & 42 & 89 & 7 & 10 & & & & \\
\hline$D M O$ & 5,719 & 5,693 & 5,751 & 92 & 5,846 & 13 & 73 & 11 & & 5,797 & 24 & 5,693 \\
\hline export & 2,971 & 3,333 & 3,461 & 3,533 & 3,596 & 3,876 & 4,104 & 4,500 & 4,754 & 4,825 & 4,238 & 4,666 \\
\hline & 938 & 3,272 & 3,361 & 3,414 & 3,526 & 3,796 & 4,029 & 4,411 & 4,672 & 4,727 & 4,153 & 4,551 \\
\hline
\end{tabular}

Source: http://appsso.eurostat.ec.europa.eu/nui/setupModifyTableLayout.do

barriers and a closer level of productivity among world business partners could advocate in favour of such outputs. For simulation purposes, different Tsallis-q parameter values were computed over the domain of definition of $q \in[1,3]$ which covers the Gaussian basin of attraction $[1<q<5 / 3]$ and Levy's attractors law $[5 / 3<q<3]$. The $q$-parameter has been incremented by a step of 0.25 starting from unity (the Shannon entropy point). These different values of $q$ generated the model error disturbances which allowed the computation of the error coefficient variation (CV). The index CV is obtained by dividing the model standard error by the average value of the dependent variable. All the above models present an error coefficient variation (CV) of around zero. The Tsallis Information Index (Bwanakare, 2014) presented in Part II of this book is around unity for the three models, suggesting relatively close to zero information divergence between priors and posteriors, under given model restrictions. We would have expected optimal solutions for $q$ less than 5/3 or, in the worst case, less than 2 for theoretical and empirical evidence. This is the case for the two commercial CET and CECS models where $q$ is almost equal to unity, suggesting a Gaussian distribution. For the CESP production model, minimum LS errors are obtained for $q$ 


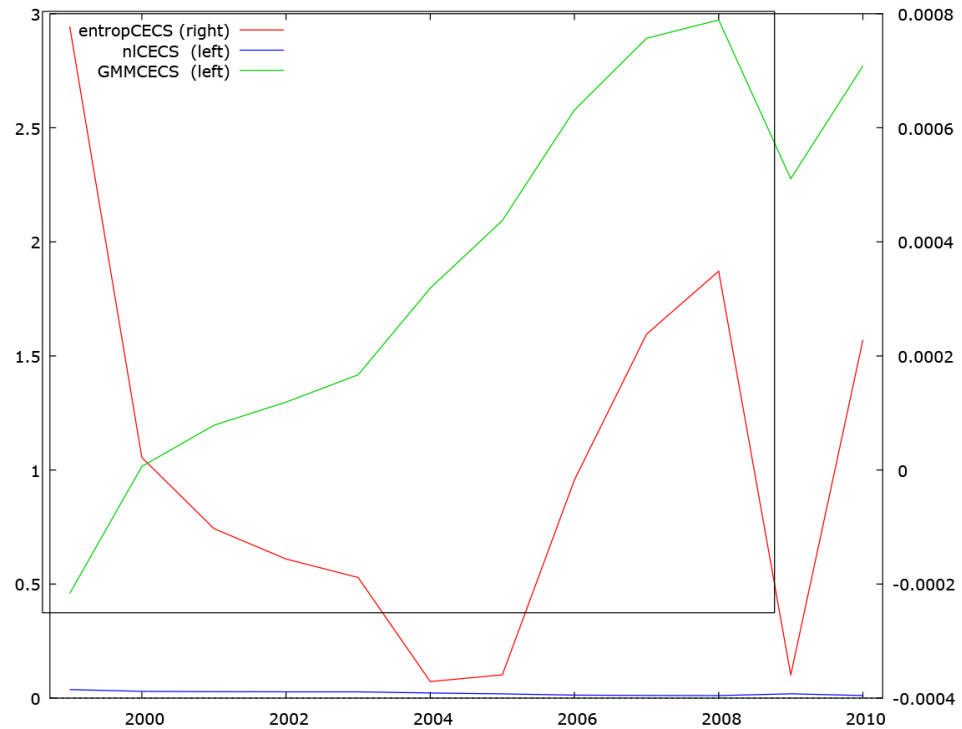

Figure 4: Error term for NLS, cross-entropy and GMM estimated models (CECS model).

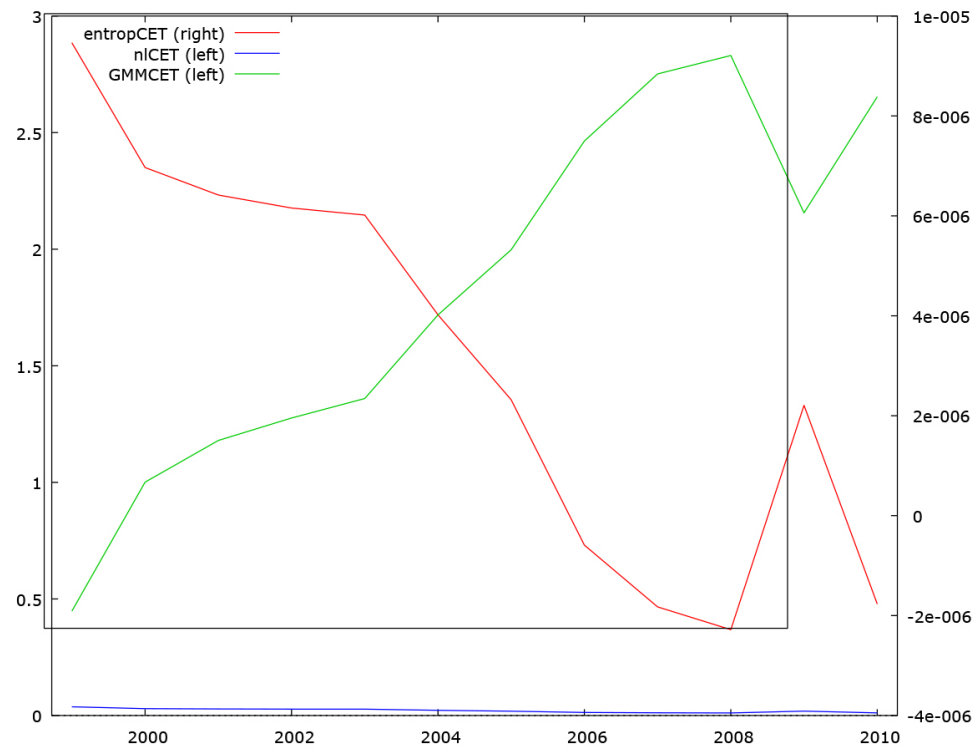

Figure 5: Error term for NLS, cross-entropy and GMM estimated models (CET model). 


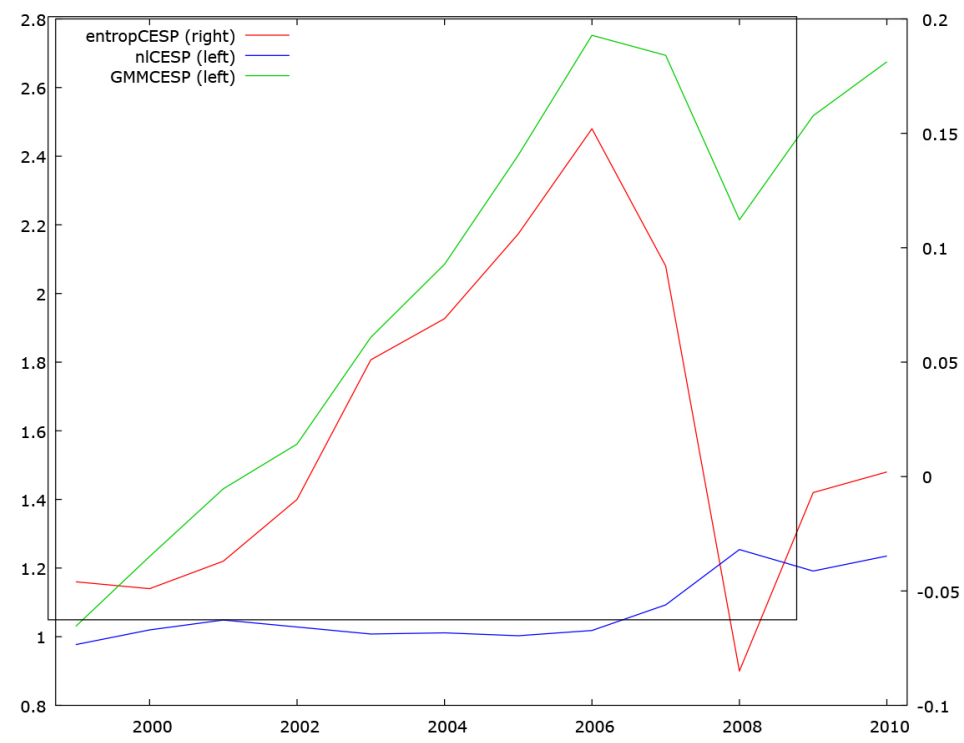

Figure 6: Error term for NLS, cross-entropy and GMM estimated models (CESP model).

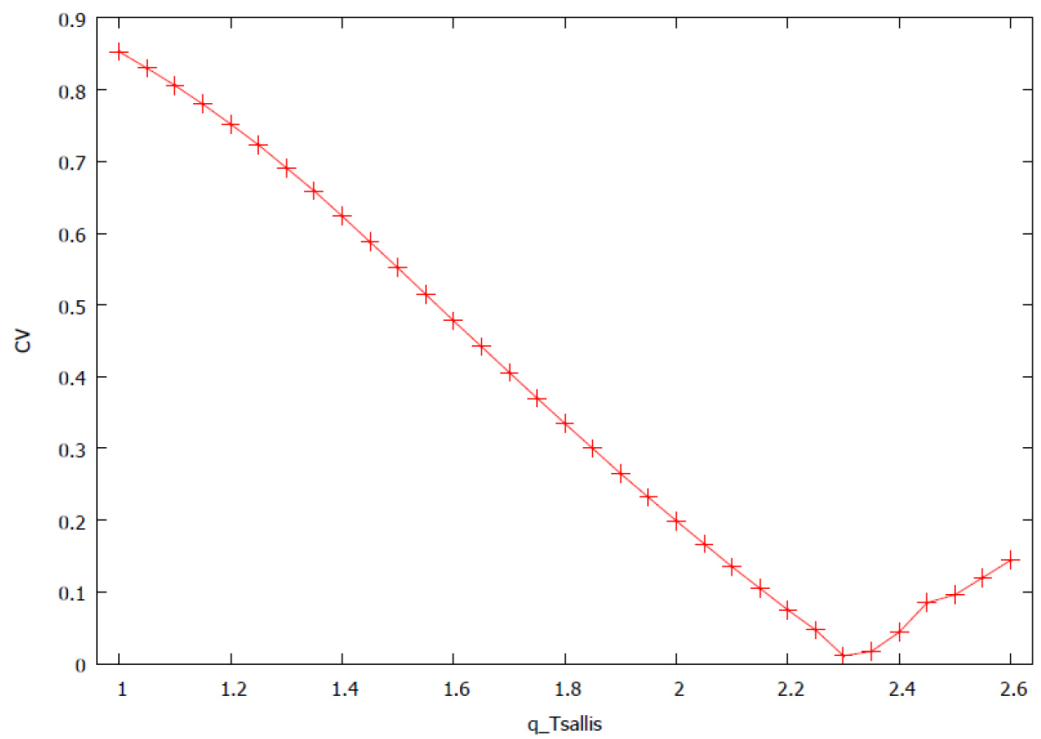

Figure 7: Model disturbance (CV) curve as a function of $q$, for $[1<q<2.6]$ (model CESP). 


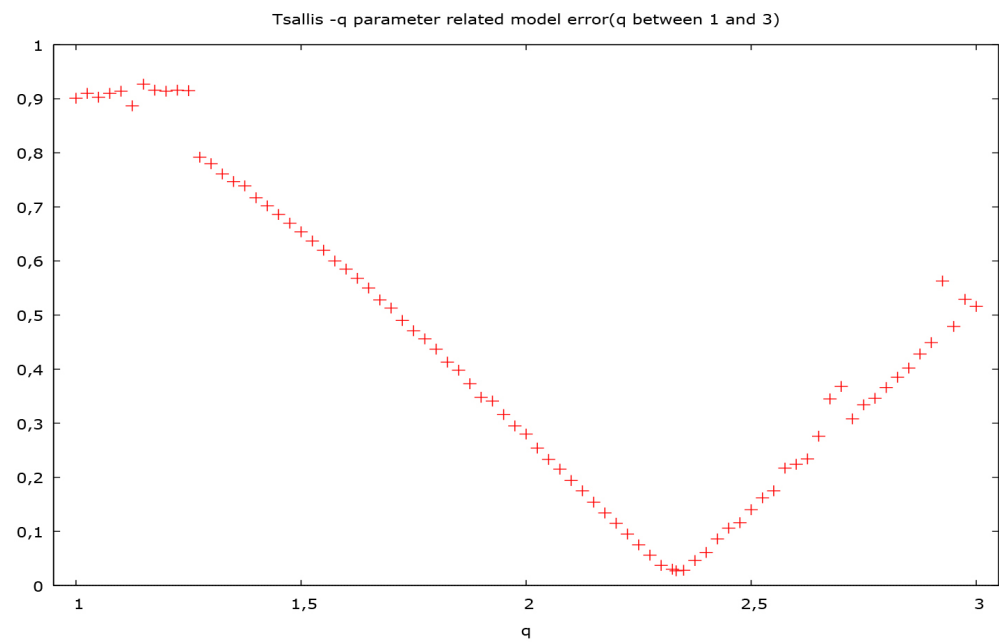

Figure 8: The Tsallis q-parameter related model error for the Belgium model (q between 1 and 3).

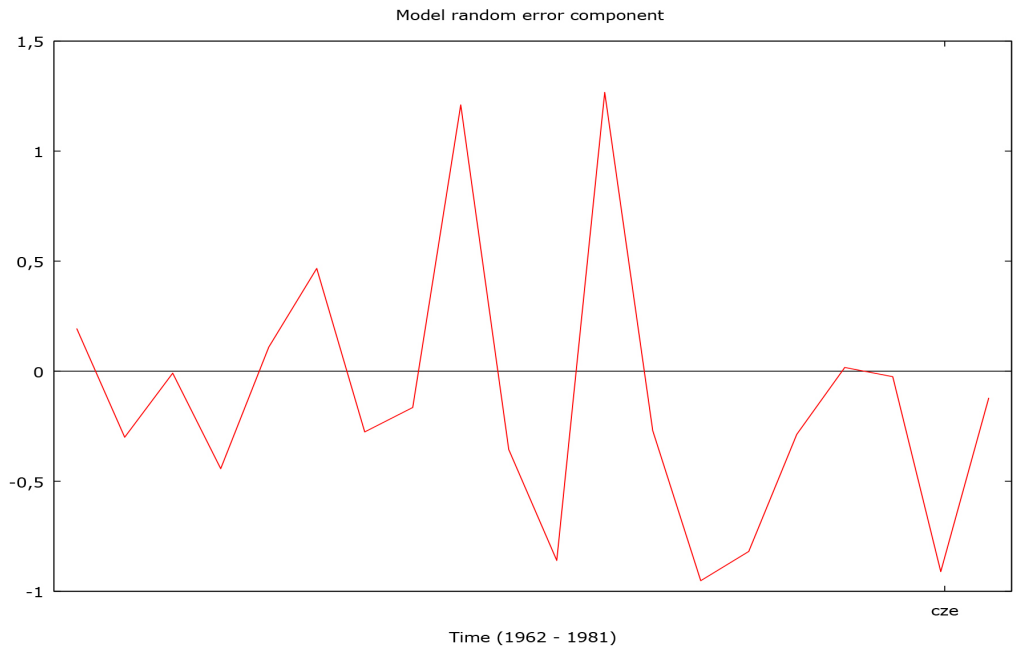

Figure 9: Model random error coefficient for SIC 33: the USA Primary Metals production NCEE model.

around 7/3 (see Figure 6). However, comparable outputs from Borges (2004) exist. He found cumulative distribution of the scaled gross domestic product of 167 countries around the world for the year 2000 corresponding to $q=3.5$. Figure 7 and 8 display a convex space defining different optimal CV values owing to different simulated $q$-Tsallis parameters for the CESP model. Minimum CV corresponds to the minimum of information divergence or of the sum of geometrical error of least squares. To verify the Tsallis related model outputs, we have computed a classical S-K-L cross-entropy econometric model which has produced for all the three models, as expected, the 
same values as those obtained from Tsallis formalism for $q$ equal to unity. Such trivial results have not been reported in the above output tables. In the case of the CESP model, we found for $q$ converging to unity a CV of $85.3 \%$ in the case of K-L, against $0.06 \%$ for an optimal $q$ equal to 2.331 . Thus, this point shows the advantage of modelling with non-extensive entropy rather than Shannon entropy, reduced to $q$ equal to unity. Finally, in Tables 20 and 21 model outputs are limited to two CESP outputs of Belgium and the USA Primary Metals production model. Contrary to all the models that have been presented in this section, this last USA study treats a space model, already discussed in Green ${ }^{57}$. As expected, the outputs from the NCEE reveal a higher precision than those from the NLS approach. In spite of a high coefficient of determination displayed $R^{2}$, economic theory related signs and space area of parameters remain inappropriate in the case of NLS outputs.

\subsection{Conclusions}

The present example has presented the rationale of the proposed Tsallis cross-entropy approach for unstable, nonlinear econometric models in a more elegant way than in the preceding applications. Though the experiment was limited to three different CES models with respect to their stochastic forms, a large class of economic and financial models could fall into this category. Only outputs produced by Tsallis formalism reflect these stochastic differences a priori known. The Tsallis entropy super-convergence estimator should only be explained, even in this unique but complex experiment, by the data generating $\mathrm{Pl}$ distribution. More investigation, particularly on the ARFIMA class of models, is needed to confirm the above findings and the importance of the PL approach in econometrical modelling.

\section{Bibliography - Part V}

Adelman, I., \& Robinson, S. (1978). Income Distribution Policy in Developing Countries: A Case Study of Korea. Stanford: Stanford University Press.

Armington, P.S. (1969). A theory of demand for products distinguished by place of production. IMF Staff papers 16, pp. 159-76.

Arndt C., Tarp, F., Robinson, S., Jensen, H.T., \& Cruz, A. (2001). Parameter estimation for a Computable general equilibrium model: A maximum entropy approach. Washington: Working Paper, International Food Policy Research Institute.

57 See Green (2003). 
Arndt C., Finn, T., Sherman, R., Jensen, H.T., \& Cruz, A. (1998). Social Accounting Matrices for Mozambique 1994 and 1995. Washington, D.C. 20006 U.S.A.: Working Paper, International Food Policy Research Institute.

Arrow, K.J., \& Debreu, G. (1954). Existence of an equilibrium for a competitive economy. Econometrica, 22, 265-90.

Arrow, K.J., \& Hahn, F.H. (1971). General Competitive Analysis. San Francisco: Holden Day.

Arrow, K.J., Chenery, H.B., Minhas, B.S., \& Solow, R.M. (1961). Capital-Labor Substitution and Economic Efficiency. The Review of Economics and Statistics, 43(3), pp. 225-250.

Bento, A.M., \& Jacobsen, M. (2007). Ricardian rents, environmental policy and the 'double-dividend' hypothesis. J. Environ. Econ. Manag., 53 (1), pp. 17-31.

Bottazzi G., Cefis, E., Dosi, G., \& Secchi, A. (2007). Invariances and Diversities in the Patterns of Industrial Evolution: Some Evidence from Italian Manufacturing Industries. Small Business Economics, 29(1), pp. 137-159.

Bourguignon, F., Michel, G., \& Miqueu, D. (1983). Short Run Rigidities and Long Run Adjustments in a Computable General Equilibrium Model of Income Distribution and Development. Journal of Development Economics, pp. 1-2.

Bwanakare, S. (2014). Non-Extensive Entropy Econometrics: New Statistical Features of Constant Elasticity of Substitution-Related Models. Entropy, pp. 2713-2728.

Bwanakare, S. (2016). Non-Extensive Entropy Econometrics and CES production Models: Country Case Study. Journal of the International Association for Official Statistics (IAOS). Retrieved from: http://content.iospress.com/articles/statistical-journal-of-the-iaos/sji1021

Capros, P., Karadeloglou, P., \& Mentzas, G. (1990). An empirical assessment of macroeconometric and CGE approaches in policy modeling. Journal of Policy Modelling, 12 (3), pp. 557-585. doi:http://dx.doi.org/10.1016/0161-8938(90)90013

De Melo, J. (1988). Computable General Equilibrium Models for Trade Policy analysis in Developing Countries: A Survey. Journal of Policy Modeling, 4, pp. 469-503.

Decaluwe, B., \& Martens, A. (1988) CGE Modeling and Developing Economies: A Concise Empirical Survey of 73 Application to 26 Countries. Journal of Policy Modeling 10:4.

Decaluwé, B., Martens, A., \& Savard, L. (2001). La politique économique du développement et les modèles d'équilibre général calculable. Montréal, Canada: Les Presses de l'Université de Montréal.

Devarajan, S., \& Robinson, S. (1983). Selected Bibliography on Computable General Equilibrium Models of Developing Countries. Harvard and U.C. Berkeley: Mimeo.

McDonald, S., Thierfelder, K., Robinson, S. (2007). Globe: A SAM Based Global CGE Model using GTAP Data. Retrieved from www.usna.edu/EconDept/RePEc/usn/wp/usnawp14.pdf

Evans, G.W., \& Ramey, G. (2006). Adaptive Expectations, Underparameterization and the Lucas Critique. Journal of Monetary Economics, 53, pp. 249-264.

Francois, J. (2001). Flexible Estimation and Inference Within General Equilibrium Systems. Discussion Paper. 0129. Adelaide 5005, Australia: Adelaide University.

Frasera, L., \& Waschik, R. (2013). The Double Dividend hypothesis in a CGE model: Specific factors and the carbon base. Energy Economics, 39, pp. 283-295.

Gabaix, X. (2008). Power laws in economics and finance. Retrieved from: http://www.nber.org/papers/w14299

Giffin, A. (2009). From Physics to Economics: An Econometric Example Using Maximum Relative Entropy. Physica A: Statistical Mechanics and its Applications, pp. 1610-1620.

Ginsburg, V., \& Waelbroeck, J. (1981). Activity Analysis and General Equilibrium Modelling, Amsterdam North-Holland. Amsterdam: North-Holland.

Ginsburgh, V., \& Keyzer, M. (1997). Structure of Applied General Equilibrium Models, MIT Press. MIT Press. 
Ginsburgh, V., \& Waelbroeck, J. (1976). Computational Experience with a Large General Equilibrium Model in computing equilibria. Amsterdam North-Holland.

Go, D.S., Lofgren, H., Ramos, F.M., \& Robinson, S. (2014). Estimating Parameters and Structural Change in CGE Models Using a Bayesian Cross-Entropy Estimation Approach. Retrieved from https://www.gtap.agecon.purdue.edu/resources/download/7136.pdf

Go, D.S., Lofgren, H., Ramos, F.M., \& Robinson, S. (2015). Estimating parameters and structural change in CGE models using a Bayesian cross-entropy estimation approach. Policy Research working paper no. WPS 7174.

Golan, A., Karp, L.S., \& Perloff, J.M. (1996). Esimating a mixed strategy employing maximum entropy. Working paper, California Agricultural Experiment Station. California.

Golan, A., Judge, G., \& Miller, D. (1996). Maximum Entropy Econometrics: Robust Estimation with Limited Data, Chichester, England: Wiley.

Gossen, H.H. (1983). The laws of human relations and the rules of human action derived therefrom. Cambridge: MIT Press.

Green, W. (2003). Basics of Econometrics. NY: Hall Prencite, 5th edition.

Guerrien, B. (2000). Dictionnaire d'analyse économique. Paris, France: La Decouverte.

Harberger, A.C. (1959). The Corporation Income Tax: An Empirical Appraisal. Tax Revision Compendium 1, pp. 231-240.

Harberger, A.C. (1962). The Incidence of the Corporation Income Tax. Journal of the Political Economy. 70, pp. 215-240.

Harris, R., \& Cox, D. (1983). Trade, Industrial Policy and Canadian manufacturing. Canadian journal of economics.

Herault, N. (2006, March). Building And Linking A Microsimulation Model To A CGE Model For South Africa. South African Journal of Economics, 74(1), pp. 34-58.

Ikeda, Y., \& Souma, W. (2008). International Comparison of Labour Productivity Distribution. (Cornell University Library) Retrieved from arXiv:0812.0208v4

Jaynes, E.T. (1988). Maximum-entropy and Bayesian methods in Science and Engineering, Vol. One: Foundations. Boston, USA: G.J. Erickson and C.R. Smith, Kluwers Accademic Publishers.

Jevons, W.S. (1879). The Theory of Political Economy. London: Macmillan.

Jian, X. (2000). An Environmentally Extended Social Accounting Matrix, Environmental and Resource Economics. 16(4), pp. 391-406.

Jorgenson, D.W. (1984). Econometric Methods for General Equilibrium Analysis In Applied General Equilibrium Analysis. Cambridge: H. Scarf and J. Shoven, Eds., Cambridge University Press.

Jorgenson, D.W. (1998a). Growth. Volume 1: Econometric General Equilibrium Modeling. Cambridge: The MIT Press.

Judge, G.G. \& Mittelhammer, R.C. (2012). An Information Theoretic Approach to Econometrics. Cambridge, UK: Cambridge University Press.

Klump, R., \& Papageorgiou, C. (2008). The CES Production Function in the Theory and Empirics of Economic Growth. Journal of Macroeconomics, 2, pp. 599-600.

Kullback, S., \& Leibler, R.A. (1951). On information and sufficiency. Annals of Mathematical Statistics, 22, 79-86.

Kydland, F.E., \& Prescott, E.C. (1982). Time to Build and Aggregate Fluctuations. Econometrica, 50 (6), pp. 1345-1370. doi:10.2307/1913386. JSTOR 1913386

Kydland, F., \& Prescott, E.C. (1977). Rules Rather than Discretion: The Inconsistency of Optimal Plans. Journal of Political Economy, pp. 473-492. doi:10.1086/260580

Leontief, W. (1941). The Structure of American Economy, 1919-1929. Cambridge: Harvard University Press.

Lofgren, H., Harris, R.L., \& Robinson, S. (2000). A Standard Computable General Equilibrium (CGE) Model in GAMS. Washington: Intl Food Policy Res Inst. 
Lucas, R. (1976). Econometric Policy Evaluation: A Critique. 1, 19-46. doi:10.1016/ S0167-2231(76)80003-6

Mansur, A., \& Whalley, J. (1984). Numerical Specification of Applied General Equilibrium Models: Estimation, Calibration, and Data in Scarf and Shoven. Applying General Equilibrium Analysis, pp. 69-127.

Mantegna, R.N., \& Stanley, H.E. (1999). Introduction to Econophysics: Correlations and Complexity in Finance. Cambridge: Cambridge University Press.

Marshall, A. (1890). Principles of Economics. London: Macmillan.

McKenzie, L.W. (1959). On the Existence of General Equilibrium for a Competitive Economy. Econometrica, 1, pp. 54-71. doi:10.2307/1907777. JSTOR 1907777

McKenzie, L.W. (1981). The Classical Theorem on Existence of Competitive Equilibrium. Econometrica, 4, pp. 819-841. doi:10.2307/1912505. JSTOR 1912505

McKenzie, L.W. (1987). Turnpike theory. The New Palgrave: A Dictionary of Economics, pp. 712-720.

McKenzie, L.W. (1954). On Equilibrium in Graham's Model of World Trade and Other Competitive Systems. Econometrica, 2, pp. 147-161.

Muth, J.F. (1961). Rational Expectations and the Theory of Price Movements. Econometrica, 3, pp. 315-335. doi:10.2307/1909635

Nash, J.F. (1950). The Bargaining Problem. Econometrica, 2, pp. 155-162. doi:10.2307/1907266. JSTOR1907266

Pearce, D. (1991). The Role of Carbon Taxes in Adjusting to Global Warming. The Economic Journal (101), pp. 938-948.

Pukelsheim, F. (1994). The Three Sigma Rule. American Statistical Association, 2, pp. 88-91.

Pyatt, G., \& Thorbecke, E. (1976). Planning Techniques for a Better Future. Geneva, Switzerland: International Labour Office.

Pyatt, G. (1988). A SAM approach to modeling. Elsevier, Journal of Policy Modeling, 10(3), pp. 327-352.

Pyatt, Graham, \& Roe, A. (1977). Social Accounting Matrices for Development Planning: With Special Reference to Sri Lanka. Cambridge: Cambridge University Press.

Sasmaz, M.U. (2016). Validity of double dividend hypothesis in EU-15 countries. Global Journal on Humanities \& Social Sciences, pp. 30-36.

Scarf, H. (1984). The Computation of Equilibrium Prices. Cambridge: (H. Scarf and J. Shoven, Eds.), Cambridge University Press.

Scarf, H. (1969). An Example of an Algorithm for Calculating Equilibrium Prices. American Economic Review, 59.

Shoven, J.B., \& Whalley, J. (1972). A General Equilibrium Calculation of the Effects of Differential Taxation of Income from Capital in U.S. Journal of Public Economics 1 (3-4), pp. 281-321.

Shoven, J.B., \& Whalley, J. (1992). Applying General Equilibrium. New York: Cambridge University Press.

Sims, C.A. (1987). A Rational Expectations Framework for Short-Run Policy Analysis. New Approaches to Monetary Economics, pp. 293-308.

Sims, C.A., 1987. A rational expectations framework for short-run policy analysis. In: Barnett, W.A., Singleton, K.J. (Eds.), New approaches to monetary economics. Cambridge University Press, Cambridge, UK, pp. 293-308.

Sims, C.A. (2007). Bayesian Methods in Applied Econometrics, or, Why Econometrics Should Always and Everywhere Be Bayesian. Princeton : Princeton University.

Stone, R. (1955). Input-Output and the Social Accounts. New York: J. Wiley.

Stone, R., \& Brown, A. (1962). A computable model for economic growth. Cambridge, UK: Cambridge Growth Project. 
Taheripour, F., Khanna, M., \& Nelson, C.H. (2008). Welfare impacts of alternative public policies for agricultural pollution control in an open economy: a general equilibrium framework. $\mathrm{Am}$. J. Agric. Econ., 90(3), pp. 701-718.

Takeda, S. (2007). The double dividend from carbon regulations in Japan. J. Jpn. Int. Econ., 21, pp. 336-364.

Tovar, C. (2009). DSGE Models and Central Banks. Economics, 3 (2009-16)(1). doi:10.5018/economics-ejournal.ja.2009-16

Tsallis, C. (2009). Introduction to Nonextensive Statistical Mechanics: Approaching a Complex World. Berlin: Springer.

Walras, L. (1874). Éléments d’économie politique pure, ou, Théorie de la richesse sociale. Lausanne: L. Corbaz \& Cie. 
